\title{
Research on Double Inverted Pendulum System Base on Hierarchical Fuzzy Sliding-mode Decoupling Control Method
}

\author{
Yinyin Zhao \\ Engineering Training Center of Beihua University, Jilin China \\ zhyy8119@126.com
}

Keywords: Hierarchical Fuzzy sliding-mode control; Decoupling control; Double inverted pendulum Introduction

\begin{abstract}
Hierarchical fuzzy sliding-mode decoupling control method is adapted in this paper, and the nonlinear system of a double inverted pendulum is decoupled into several subsystems, an adaptive law based on the Lyapunov function is used to tune the coupling factor of the hierarchical sliding-mode controller, a favorable decoupling performance is achieved under the condition of the system guaranteed stability by simulation experiments. Simulations are performed and demonstrated the feasibility of the proposed design method, which has been more predominant than adopting conventional fuzzy sliding-mode decoupling control in decoupling performance.
\end{abstract}

\section{Introduction}

Research has important practical significance which in the uncertain condition nonlinear system control problem. Sliding-mode control is the effective method to solve the problem of nonlinear, but there are sliding mode switching litter problem ${ }^{[1][2]}$; Fuzzy sliding-mode control can reduce jitter, but sometimes can appear unstable phenomenon for the strong coupling system ${ }^{[3]}$. In this paper, combining with the research puts forward the hierarchical fuzzy sliding mode design method of decoupling control. First, a class of nonlinear coupled system is decomposed into several subsystems, and define sliding surface for each subsystem. Then, using hierarchical fuzzy model controller to control the whole system, will not only ensure the decoupling system performance, but also has good stability and robustness characteristics.

\section{Double Inverted Pendulum System Model}

In this study, the control device is Googol Technology Company's linear double pendulum system. Linear double pendulum's schematic diagram as shown in fig. 1.

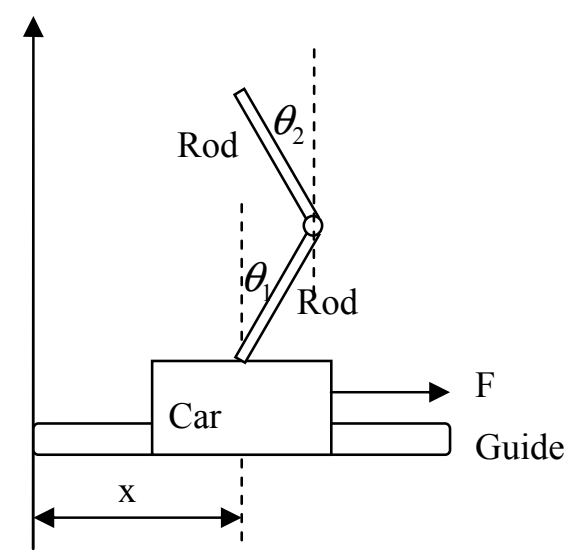

Figure 1. Structure of double inverted pendulum 
It consists of guide rail, car, and two pendulums. A torque motor at one end of the guide rail, motor drive the car slides along the guide rail. Double inverted pendulum control system in the servo motor; pendulum rod 1 and pendulum pod 2 are installed on the optical encoder. Control of the double pendulum need 6 state variables, respectively are the pendulum position, pendulum speed, pendulum rod 1 position, pendulum rod 1 speed, pendulum rod 2 position, pendulum rod 2 speed. Obtained by the differential equation computing pendulum speed, a pendulum rod 1and rod 2 speed. Then use state feedback to control the inverted pendulum. Car and two pendulum rod force is transmitted via a hinge heritability, the final steady-state performance of the system will not fall or sudden shock divergence ${ }^{[4]}$.

\section{Hierarchical Fuzzy Sliding-mode Control Design}

Single-input Multi-output Nonlinear System State Description. Generally the single-input multi-output nonlinear coupled system can be described as ${ }^{[5]}$ :

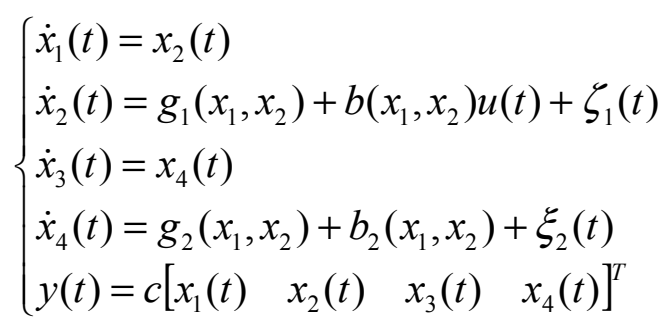

Where $x_{1}(t), x_{2}(t), x_{3}(t)$ and $x_{4}(t)$ are the state variables; $g_{1}\left(x_{1}, x_{2}\right), g_{2}\left(x_{1}, x_{2}\right), b_{1}\left(x_{1}, x_{2}\right)$ and $b_{2}\left(x_{1}, x_{2}\right)$ are limited in the name of the nonlinear; $u(t)$ is the control input; $\zeta_{1}(t), \zeta_{2}(t)$ are the comprehensive interference, and assume that they are bounded. Using hierarchical sliding mode control, define its control of the sliding-mode surface ${ }^{[6]}$ expressed as:

$$
\begin{aligned}
& s_{1}=\dot{x}_{1}+\lambda_{1} x_{1}=x_{2}+\lambda_{1} x_{1} \\
& s_{2}=\dot{x}_{3}+\lambda_{2} x_{3}=x_{4}+\lambda_{2} x_{3}
\end{aligned}
$$

Where, $\lambda_{1}$ and $\lambda_{2}$ are positives constant; by Eq.1 and Eq.2, according to the sliding-mode control condition of $\dot{s}=0$, we can get by $\left(x_{1}, x_{2}\right)$ and $\left(x_{3}, x_{4}\right)$ composed of independent control law.

Hierarchical Fuzzy Sliding Mode Control. For the above-mentioned system, the decoupled sliding-mode surface is defined as ${ }^{[7]}$ :

$$
s_{r}=s_{1}-k^{*} s_{2}
$$

Where, $k^{*}$ is the best coupling coefficient, its reaction coupling effect between the sliding-mode surfaces $s_{1}$ and $s_{2}$. In order to improve the decoupling performance of the coupled system, can be estimated by fuzzy inference $k^{*}$.

Fuzzy Inference System:

$\mathrm{R}_{\mathrm{i}}$ : If $\mathrm{s}_{\mathrm{r}}=\mathrm{A}_{\mathrm{i} 1}$ and $\mathrm{s}_{\mathrm{r}}=\mathrm{A}_{\mathrm{i} 2}$, then $\mathrm{k}_{\mathrm{fx}}=\mathrm{H}$. Where, $\mathrm{R}_{\mathrm{i}}$ is the $\mathrm{i}$-th fuzzy rule $\mathrm{i}=1,2 \cdots, \mathrm{m} ; \mathrm{H}_{\mathrm{i}}$ is described by the single adjustable volume $\phi_{i}$ fuzzy evaluation; $A_{i j}$ by the $\mathrm{j}$ input variables and $\mathrm{i}$ fuzzy rules to determine, and described by the membership function $\mu_{A_{i j}}(\cdot)$,Choose

$$
\mu_{A_{i j}}\left(x_{j}\right)=e^{\ln (0.5)\left(w_{s} x_{j}-w_{c}\right)^{2} w_{d}^{2}}
$$

$\mathrm{W}_{\mathrm{c}}$ is the Gaussian membership function center parameter value, $\mathrm{W}_{\mathrm{d}}$ is the reciprocal from the center of this value, $\mathrm{W}_{\mathrm{s}}$ used to measure $x_{i j}$.

Because $k^{*}$ is positive, so the evaluation value $\hat{k}_{f z}$ is also limited to a positive number, can be written as: 


$$
\hat{k}_{f z}\left(s_{r}, \dot{s}_{r}, \hat{\phi}\right)=\psi^{T} \hat{\phi}
$$

Where, $\hat{\phi}=\left[\hat{\phi} \hat{\phi}_{2} \cdots \hat{\phi}_{m}\right]^{T}$ is the parameter vector; $\psi=\left[\psi_{1} \psi_{2} \cdots \psi_{m}\right]^{T}$ is regression parameter vector, and $\psi$ is defined as:

$$
\psi_{i}=\frac{\zeta_{i}}{\sum_{i=1}^{m} \zeta_{i}}
$$

Where, $\zeta_{i}=\mu_{A_{i j}}\left(s_{r}\right) \cdot \mu_{A_{i j}}\left(\dot{s}_{r}\right)$ is the $\mathrm{i}$-th fuzzy rule evaluation level; $\mu_{A_{i j}}\left(s_{r}\right)$ is the fuzzy membership function. According to the law of universal approximation ${ }^{[5]}$, then there is the optimal fuzzy logic system $k_{f z}^{*}\left(s_{r}, \dot{s}_{r}, \phi^{*}\right)$ as:

$$
\begin{aligned}
& k_{f z}^{*}\left(s_{r}, \dot{s}_{r}, \phi^{*}\right)=\psi^{T} \phi^{*} \\
& \phi^{*}=\underset{\|\dot{\phi}\| \in \Omega_{\varphi}}{\arg \min }\left\{\sup _{s_{r} \in \Omega_{r}, \dot{s}_{i} \in \Omega_{\dot{r}}}\left[\hat{k}_{f z}\left(s_{r}, \dot{s}_{r}, \hat{\phi}\right)-k^{*}\right]\right\}
\end{aligned}
$$

(8)

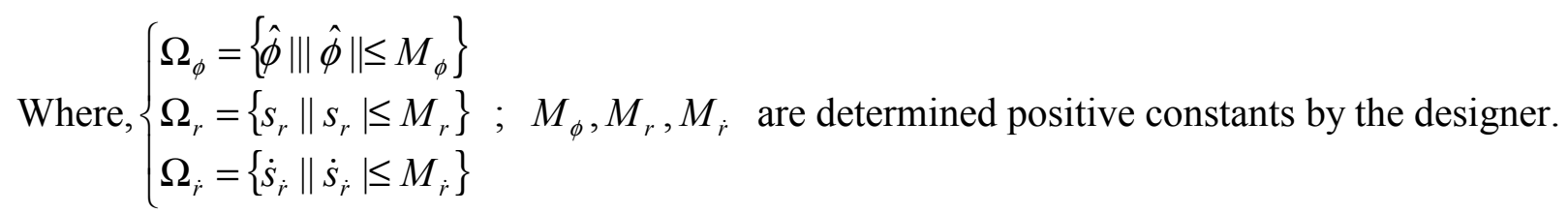

The best parameter vector of the estimation error is:

$$
\eta=\hat{\phi}-\phi^{*}
$$

Define the Lyapunov Function:

$$
V\left(s_{r}, \eta\right)=\frac{1}{2} s_{r}^{2}+\frac{1}{2 \alpha} \eta^{T} \eta
$$

Where, $\alpha$ is a positive constant. According to the stability condition choose $\eta$. The parameter vector is retained in the interior of the constraint condition.

Define the function $E(t) \equiv\left(\varepsilon-\xi_{\max }\right) s_{r}(t) \leq-\dot{V}(t)$, obtain as:

$$
\int_{0}^{t} E(t) d \tau \leq V\left(s_{r}(0), \eta(0)\right)-V\left(s_{r}(t), \eta(t)\right)
$$

Formula Eq.9 to the right is limited, so as: $\lim _{t \rightarrow \infty} \int_{0}^{t} E(t) d \tau<\infty$. Because $\dot{E}(t)$ is limited, so $E(t)$ continuously. Use Barbalat lemma, can be obtained: $\lim _{t \rightarrow \infty} E(t)=0$, so $s_{r}(t) \rightarrow 0$.

So, we can get control rule:

$$
u=u_{e g}+u_{n}=\left(\hat{k}_{f 2} \dot{s}_{2}-\varepsilon_{1} \operatorname{sgn}\left(s_{r}\right)-\delta \operatorname{sgn}\left(s_{2} s_{r}\right) \dot{s}_{2}-g_{1}-\lambda_{1} x_{2}\right) / b_{1}
$$

\section{Simulation Results}

Linear double inverted pendulum system selection as follows: $m_{1}=m_{2}=m=1 \mathrm{~kg}$, pendulum length are $0.12 m, g=9.8 m / s^{2}, \xi=0.05^{\circ} / s^{2}$, Gaussian membership function center parameter value 
$w_{c}=[-1.5,-0.5,0,0.5,1.5]$, set $w_{s}=1$ and $w_{d}=0.5$. The initial value of $\hat{\phi}_{i}$ and $\dot{\hat{\phi}}$ are 0.1 , the initial condition are $x_{1}=30^{\circ}, x_{2}=0^{\circ} / s, x_{3}=10^{\circ}, x_{4}=0^{\circ} / s, x_{5}=0 m, x_{6}=0 m / s, i=1,2, \cdots, 25$. For hierarchical fuzzy sliding-mode control, when $\lambda_{1}=5.5, \lambda_{2}=0.5$ and $\lambda_{3}=0.1$, to establish three hierarchical sliding-mode surfaces $s_{1}, s_{2}, s_{3}$. When $\alpha=\bar{\alpha}=0.05, \quad \delta_{1}=\delta_{2}=0.5$ and $\varepsilon_{1}=5$, adjust the optimum coupling coefficient $\hat{k}_{f z 1}$ and $\hat{k}_{f z 2}$. When the value of $\hat{k}_{f z 1}=0.017$ and $\hat{k}_{f z 2}=0.672$, hierarchical fuzzy sliding-mode decoupling control and fuzzy sliding-mode decoupling control of the simulation results are shown in fig. 2. Simulation results show that by using hierarchical fuzzy sliding-mode decoupling control, all of the states assemble in zero places has the good performance of the system.

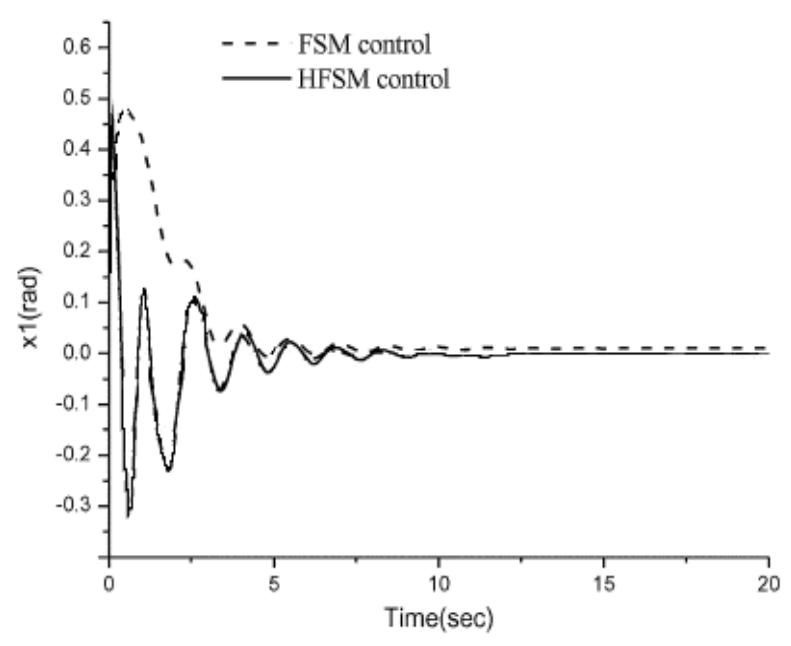

Figure 2. Angle1 curve of double inverted pendulum

\section{Conclusion}

Hierarchical fuzzy sliding mode control of single-input multiple-output decoupling of nonlinear coupled system provides a decoupling control method. This paper is based on Lyapunov function, constitute the hierarchical fuzzy sliding-mode controller. By adjusting the decoupling coefficient to adapt to the rules, on the premise of guarantee the stability of system, improve the coupling nonlinear system decoupling performance. For double inverted pendulum system, the simulation results show that the control method is feasible and has good control performance. And for general nonlinear coupling system research provides a new theoretical method.

\section{References}

[1] A.L. Li, H.Y. Qi and L. Liang: 2014 2nd International Conference on Manufacturing Engineering and Technology for Manufacturing Growth(Miami,State of Florida,USA, 2014).Vol. 1, p.306.

[2] Y.D. Wang, H.Y. He, W.F. Shi and W.F. Zhang: 2012 Third International Conference on Theoretical and Mathematical Foundations of Computer Science( Bali,Indonesia, 2012).Vol. 38, p.555.

[3] J.L. Zhang, W. Zhang: Computer Simulation, Vol. 10, p.341.(In Chinese)

[4] A.Banerjee, Y.Arkun, B.Ogunnaike and R.Pearson: AIChE J, 5(2013), p.102.

[5] S.J. Mou, F. Liu: 2015 IEEE Advanced Information Technology, Electronic and Automation Control Conference(Chongqing, China, 2015). Vol. D, p.395. 
[6] Z.J. Yang, Y.H. Shi: The 2016 International Conference on Advanced Materials, Structures and Mechanical Engineering (Incheon,Korea, 2016).Vol. 1, p.37.

[7] P.Bhavsar, V.Kumar: International Journal of Intelligent Systems and Applications(IJISA), 3(2012), p.31. 